\title{
The Impact of Dyslipidemia on the Health-Related Quality of Life of Korean Females Aged 50 Years and Older
}

\author{
*Hyejin PARK \\ Department of International Healthcare Management, Daegu Catholic University, Kyungbuk, Republic of Korea \\ *Correspondence: Email: hjpark@cu.ac.kr
}

(Received 10 Apr 2018; accepted 10 May 2018)

\section{Dear Editor-in-Chief}

Dyslipidemia is one of the major risk factors of cardiovascular disease by contributing to the development and progression of atherosclerosis (1). In Korea, $47.8 \%$ of people over $30 \mathrm{yr}$ of age had dyslipidemia in 2015 and the prevalence of dyslipidemia is steadily increasing (2). Particularly, the prevalence of dyslipidemia in women increases significantly after the age of 50 (2). Dyslipidemia has negative effect on health-related quality of life (HRQoL) (3), even though relatively few studies have measured the HRQoL associated with dyslipidemia in women aged $50 \mathrm{yr}$ and over.

To evaluate HRQoL in the target population, many tools, including Medical Outcome Study Short Form-36 (SF-36), EuroQol-5 Dimension (EQ-5D), and Health Utility Index Mark 3 (HUI3), were developed (4). Among these instruments, EQ-5D is a self-reported questionnaire that consists of five domains of HRQoL and standardized research tools to measure and describe HRQoL (5). Because of high validity and reliability, EQ-5D has recently been widely used in the measurement of HRQoL for patients with disease in many studies. The aim of this study was to investigate the association between dyslipidemia and HRQoL in women over $50 \mathrm{yr}$ of age using the Korea National Health and Nutrition Examination Survey (KNHANES). For this purpose, this study analyzed the relationship between prevalence of dyslipidemia and HRQoL in each of the 5 dimensions of EuroQoL.
This study was undertaken using data from the KNHANES 2014-2015, which included a health and nutrition survey and a medical examination. The KNHANES sample was selected using a stratified multistage cluster sampling design with proportional allocation based on the National Census Registry. From this sample, 3198 women ( $\geq 50 \mathrm{yr}$ of age) provided data without values missing for any of the variables analyzed in this study. Participants were asked their demographic characteristics and presence of dyslipidemia. Height and weight were measured with the subjects wearing light clothing and no shoes. BMI was then calculated as weight $(\mathrm{kg})$ divided by the square of height $\left(\mathrm{m}^{2}\right)$. The subjects were categorized as underweight $(\mathrm{BMI}<18.5)$, normal $(18.5 \leq \mathrm{BMI}<22.9), \quad$ overweight $\quad(23.0 \leq$ $\mathrm{BMI}<24.9)$, and obese (BMI $\geq 25.0)$ according to the WHO definitions for Asian populations (6). The study protocol was approved by the Korean Ministry of Health and Welfare and was conducted in accordance with the Ethical Principles for Medical Research Involving Human Subjects, as defined by the Helsinki Declaration. The study participants provided written informed consent. HRQoL was assessed using EQ-5D questionnaire. EQ-5D is a self-reported descriptive system instrument with five health dimensions (mobility, self-care, pain/discomfort, usual activities, and anxiety/depression) each divided into three different levels, namely no problems, some or 
moderate problems and severe or extreme problems (7). The level scores were used in order to describe an overall measure of perceived HRQoL.

Logistic regression models were used to estimate the odds ratio (OR) and 95\% CIs for abnormal (disability) versus normal (no problem) in the categories of EuroQol among participants who reported to have dyslipidemia compared with the reference group (those who reported to not have dyslipidemia). All statistical analyses were con- ducted using SAS version 9.4 (SAS Institute, Cary, NC, USA).

The mean age and BMI of participants without dyslipidemia were 63.6 and 24.0, respectively. In contrast, the mean age and BMI were 65.6 and 24.4, respectively, in the subjects with dyslipidemia. The EQ-5D index was lower in participants with dyslipidemia (with dyslipidemia: $0.87 \pm 0.18$, without dyslipidemia: $0.89 \pm 0.16, P<0.001)$ suggesting significant impairment of HRQoL in the elderly women with dyslipidemia (Table 1).

Table 1: Age, BMI, and EQ-5D of study population by category of dyslipidemia

\begin{tabular}{lccc}
\hline Variable & All & Non-dyslipidemia & Dyslipidemia \\
\hline $\mathrm{N}$ & 3198 & 2430 & 768 \\
Age $(\mathrm{yr})$ & $64.2 \pm 9.1$ & $63.6 \pm 9.4$ & $65.8 \pm 7.8$ \\
$\mathrm{BMI}\left(\mathrm{kg} / \mathrm{m}^{2}\right)$ & $24.2 \pm 3.3$ & $24.0 \pm 3.2$ & $24.9 \pm 3.3$ \\
EQ-5D & $0.89 \pm 0.17$ & $0.89 \pm 0.16$ & $0.87 \pm 0.18$ \\
Data are presented as mean \pm standard deviation & & \\
\hline
\end{tabular}

Table 2 shows the ORs for disabilities associated with dyslipidemia. The age-adjusted ORs for problems with mobility, self-care, and usual activities were not significantly associated with dyslipidemia. However, compared to those with- out dyslipidemia, those with dyslipidemia had adjusted ORs for pain/discomfort and anxiety/depression of 1.24 (95\% CI: 1.05-1.47, $P=0.014)$ and 1.37 (95\% CI: $1.12-1.68, P=0.002$ ), respectively.

Table 2: Age-adjusted odds ratios (95\% CI) for disability in the categories of EuroQol by the prevalence of dyslipidemia in Korean women $50 \mathrm{yr}$ of age or older

\begin{tabular}{lccc}
\hline EuroQoL domain & $\begin{array}{c}\text { Non-dylipidemia } \\
(\mathbf{n = 2 4 3 0 )}\end{array}$ & $\begin{array}{c}\text { Dyslipidemia } \\
(\boldsymbol{n}=\mathbf{7 6 8})\end{array}$ & $\boldsymbol{P}$ \\
\hline EuroQoL-mobility & 1.00 (reference) & $1.12(0.93-1.35)$ & 0.229 \\
EuroQoL-self care & 1.00 (reference) & $1.13(0.85-1.50)$ & 0.393 \\
EuroQoL-usual activities & 1.00 (reference) & $1.12(0.91-1.39)$ & 0.290 \\
EuroQoL-pain/discomfort & 1.00 (reference) & $1.24(1.05-1.47)$ & 0.014 \\
EuroQoL-anxiety/depression & 1.00 (reference) & $1.37(1.12-1.68)$ & 0.002 \\
\hline
\end{tabular}

Thus, this study showed that dyslipidemia significantly compromised the HRQoL of Korean females aged $\geq 50 \mathrm{yr}$. In terms of the five dimensions of the EQ-5D, the ORs for disability in the dimensions of pain/discomfort and anxiety/depression were significantly higher in Korean females with than in those without dyslipidemia. In particular, anxiety/depression exerted the strongest influence on the HRQoL of dyslipidemic subjects, suggesting that the prevention and management of anxiety/depression are important for improving the HRQoL of such individuals.

\section{Acknowledgments}

This work was supported by research grants from Daegu Catholic University in 2017.

\section{Conflict of interest}

The authors declare that there is no conflict of interests. 


\section{References}

1. Pandian A, Arora A, Sperling LS, Khan BV (2008). Targeting multiple dyslipidemias with fixed combinations--focus on extended release niacin and simvastatin. Vasc Health Risk. Manag, 4(5): 1001-9.

2. Korean Society of Lipidology and Atherosclerosis (2015). Dyslipidemia fact sheet in Korea 2015. KSLA, Korea.

3. Lalonde L, Clarke AE, Joseph L et al (2001). Health-related quality of life with coronary heart disease prevention and treatment. J Clin Epidemiol, 54(10): 1011-8.

4. Muraki S, Akune T, Oka H et al (2010). Association of radiographic and symptomatic knee osteoarthritis with health-related quality of life in a population-based cohort study in Japan: the ROAD study. Osteoartbritis Cartilage, 18(9): 1227-34.

5. Søltoft F, Hammer M, Kragh N (2009). The association of body mass index and healthrelated quality of life in the general population: data from the 2003 Health Survey of England. Qual Life Res, 18(10): 1293-9.

6. WHO expert consultation (2004). Appropriate body-mass index for Asian populations and its implications for policy and intervention strategies. Lancet, 363(9403): 157-63.

7. Dritsaki M, Petrou S, Williams M, Lamb SE (2017). An empirical evaluation of the SF-12, SF-6D, EQ-5D and Michigan Hand Outcome Questionnaire in patients with rheumatoid arthritis of the hand. Health Qual Life Outcomes, 15: 20. 